\title{
Publisher Correction to: Diffusive limit of a two-dimensional well-balanced approximation to a kinetic model of chemotaxis
}

\author{
Gabriella Bretti ${ }^{1} \cdot$ Laurent Gosse $^{1}$ (D) \\ Published online: 1 June 2021 \\ (c) Springer Nature Switzerland AG 2021
}

\section{Publisher Correction to: SN Partial Differential Equations and Applications https://doi.org/10.1007/s42985-021-00087-7}

Unfortunately, formula 4.5 is incorrect. This was a mistake by the typesetter.

The original article has been corrected.

The original article can be found online at https://doi.org/10.1007/s42985-021-00087-7.

Laurent Gosse

laurent.gosse@cnr.it

Gabriella Bretti

g.bretti@iac.cnr.it

1 Istituto Per Le Applicazioni del Calcolo, via dei Taurini, 19, 00185 Rome, Italy 